\title{
NSGA-II-Based Multi-objective Mission Planning Method for Satellite Formation System
}

\author{
Xiaowei Shao ${ }^{1}$, Zehao Zhang1, Jihe Wang ${ }^{1}$, Dexin Zhang1
}

\begin{abstract}
This study proposes a non-dominated sorting genetic algorithm-II-based multi-objective optimization method to solve the multi-objective mission planning problem for satellite formation flying system which has the ability to obtain both digital elevation map and ground moving target indicator information at the same time when certain conditions are satisfied. The 2 objectives considered in this study are maximizing total profits and maximizing numbers of completed acquisitions. Thus, the multiple-objective satellite scheduling optimization problem is formulated and solved by the proposed method. Its validity and effectiveness are verified by numerical simulations, and the results show that it can achieve better performance with respect to 2 different objectives in an overall perspective than the traditional scheduling optimization, which can consider only 1 objective.
\end{abstract}

KEYWORDS: NSGA-II method, Multi-objective mission planning, DEM and GMTI, Formation flying system.

\section{INTRODUCTION}

With the development of small satellite technology, formation flying becomes a new enabling technique for many space missions, such as virtual synthetic aperture radar (SAR), space surveillance system, etc. In this study, we focus on the interferometric SAR (InSAR) mission by using new proposed formation flying technology. Differently from traditional space mission, by satisfying certain conditions, formation flyingbased InSAR mission can perform both digital elevation map (DEM) and ground moving target indicator (GMTI) missions at the same time. Hence, to increase the system effectiveness, it is essential to propose a multiple-objective satellite scheduling optimization method to deal with the new formation flyingbased InSAR mission planning problem. In order to propose the new multi-objective mission optimization method, the relevant literature review regarding to satellite mission planning is conducted here.

In relation to traditional single satellite mission scheduling problem, Bensanna et al. (1996) studied the daily scheduling problem associated with the SPOT5 satellite and generalized the satellite image collection planning as a version of the knapsack model, in which the objective was to maximize a profit function. They proposed a Tabu search algorithm to determine the image collection schedule. Lemaitre et al. (2002) applied 4 methods to solve the simplified scheduling problem, which are greedy algorithm, dynamic programming, constraint programming, and local search method. Jang et al. (2013) provided a heuristic solution approach to solve the image collection planning problem of a Korean satellite, KOMPSAT-2.

With the development of multiple satellite system, many researchers have proposed various methods to solve optimal

\footnotetext{
1.Shanghai Jiao Tong University - School of Aeronautics and Astronautics - Distributed Spacecraft System Technology Laboratory - Shanghai - China. Author for correspondence: Xiaowei Shao | Shanghai Jiao Tong University - School of Aeronautics and Astronautics - Distributed Spacecraft System Technology Laboratory | Shanghai 200240 - China | Email: shaoxwmail@163.com

Received: 05/27/2016 | Accepted: 09/14/2016
} 
mission scheduling for multiple satellite system. Xiaolu et al. (2014) partitioned the problem into 2 sub-problems: task assignment and task merging. In task assignment, they proposed an adaptive ant colony optimization algorithm to select specific time window for each task and create a task list for each satellite. In task merging, they proposed the concept of task combination and developed a dynamic programming algorithm to find the best merging plan for each satellite. Bianchessi et al. (2007) studied the selecting and scheduling requests for the multisatellite, multi-orbit, and multi-user. An upper bounding procedure based on column generation is used to evaluate the quality of the solutions. Kim and Chang (2015) performed the optimization of constellation operation by minimizing the system response time. He et al. (2011) formulated the model of satellites observation scheduling problem with task merging and developed the simulated annealing algorithm to solve the mission scheduling problem. Gao et al. (2013) designed a framework of ant colony algorithm for remote satellite and ground integration scheduling problem in the parallel environment. Hao et al. (2013) proposed a combination of genetic and ant colony algorithms to solve the mission scheduling problem. The performance of the proposed algorithm was compared with the genetic one. Tangpattanakul et al. (2015) presented an indicator-based multi-objective local search (IBMOLS) to solve a multi-objective optimization problem. The objectives are to maximize the total profit of the selected acquisitions and simultaneously to ensure the fairness of resource sharing by minimizing the maximum profit difference between users.

As aforementioned, there are various methods to deal with single task mission planning problem with 1 satellite; however, due to the unique features brought by the new developed formation flying technology, these methods cannot solve the multiple tasks scheduling problem of formation flying mission considered in this study. Hence, the multiple objective optimization method to handle satellite formation flying system with multiple task abilities is proposed here.

In this study, we focus on the mission scheduling problem for formation flying system which has multiple task ability. Instead of single objective mission planning, 2 objectives are considered, which are total profit maximization and completed mission numbers maximization. The contribution of this study is that, to solve the unique formation flying SAR mission planning which can perform 2 different tasks at the same time, a non-dominated sorting genetic algorithm (NSGA)-II-based multi-objective mission planning optimization method with several practical constraints is proposed. The new proposed method can achieve better performance with respect to 2 different objectives in an overall perspective than traditional genetic algorithm method, which can optimize only 1 objective.

\section{PROBLEM DESCRIPTION}

This section describes the imaging process of InSAR satellite, introduces the mission scheduling problem of InSAR formation flying system, and formulates the mathematical model of the multi-objective scheduling problem.

\section{OBSERVATION CHARACTERISTICS OF SYNTHETIC APERTURE RADAR SATELLITE}

SAR satellites collect image data by its SAR sensor, which observes the Earth by sweeping mode. The observation range is composed of multiple observation strips. The length of the strip depends on observing time and its width depends on the inner and outer half angle, as illustrated in Fig. 1. For simplicity, we assume the widths of all strips are equal, and there is a small amount of overlap between different observing strips.

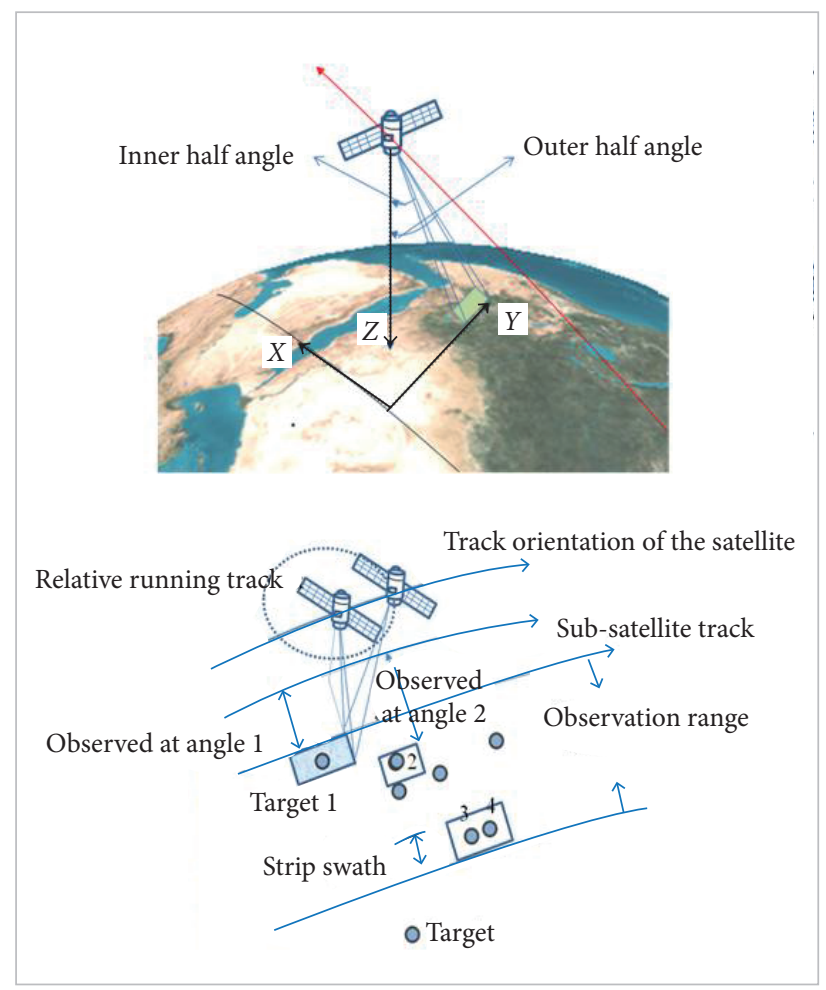

Figure 1. Observation process of the SAR satellite. 
Because the observation ranges can overlap, some targets can be observed by 2 strips, as illustrated in Fig. 2. Hence, each target can have more than 1 observation chance. With the help of orbit predicting and satellite-ground visibility computing software (Satellite Tool Kits - STK), the observation time windows between satellites and targets can be obtained as the input for the scheduling optimization algorithm, as illustrated in Table 1.

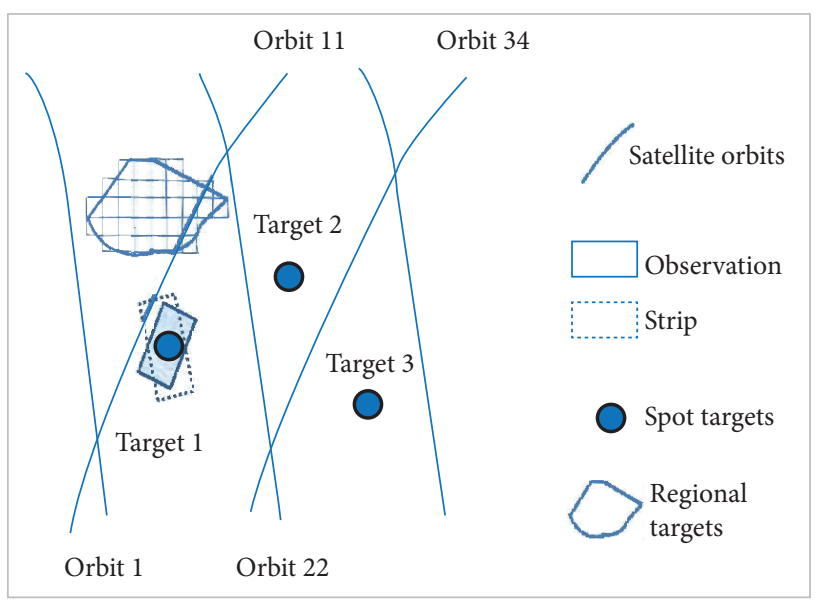

Figure 2. Satellite orbits which can observe targets.

Table 1. Observation opportunity of targets.

\begin{tabular}{|c|c|}
\hline Target & $\begin{array}{c}\text { Satellite orbits which can } \\
\text { observe targets }\end{array}$ \\
\hline 1 & 11134 \\
\hline 2 & 11122 \\
\hline 3 & 1112234 \\
\hline
\end{tabular}

\section{OBSERVATION TARGET}

Depending on the size, all the observation areas can be grouped into 2 types: point targets and regional targets. Point targets can be observed by 1 strip and the regional one needs more strips to complete region observation. Therefore, the method to decompose regional targets into multiple point ones is shown in Fig. 3, and the detail procedure of decomposition will be introduced in the next section.

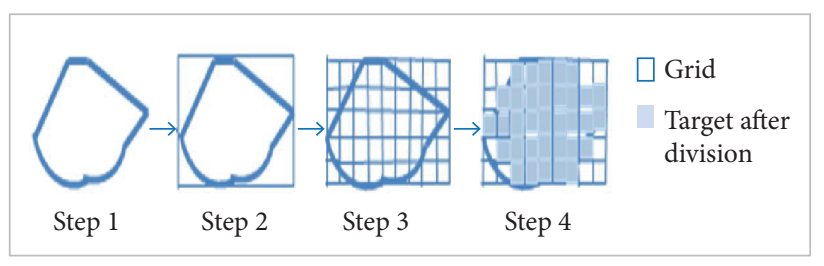

Figure 3. The steps of regional target decomposition.

\section{MULTI-OBJECTIVE DESCRIPTION}

By satisfying certain geometrical conditions, formation flying-based InSAR system can obtain DEM and GMTI images at the same time. For instance, in Fig. 1, by using strip 1, the satellite can observe target 1.The observation of targets 1 and 2 can satisfy angle switching constraints. The observing angle of targets 1 and 2 can obtain images satisfying 2 tasks (DEM and GMTI). But there is time window conflict between target 2 and targets 3 and 4 ; the observing angle of targets 3 and 4 can only obtain 1 image satisfying 1 task (DEM). If we choose to observe targets 3 and 4 , we can complete 2 targets observation. If we choose to observe target 2 , we can only obtain 1 GMTI image. Thus, we need to choose the target to be observed on the basis of objective and constraints. This study studies the multi-objective mission scheduling optimization problem of InSAR formation flying system. The constraints considered here include the maximum number of observation in 1 orbit, the longest observation time in 1 orbit, and the time to switch from a strip to another; the 2 optimization objectives are maximizing the total profit of the selected acquisitions and the number of finished acquisitions.

\section{MATHEMATICAL FORMULATION}

Based on the above discussion, the mathematical model of multi-objective mission planning is established and represented by the operator $(O, A, T, C, F)$, where $O$ is the set of orbit numbers of satellites, $O=\left\{1,2, \ldots, N u m_{-} O\right\} ; A$ is the set of strips of each orbit, $A=\left\{1,2, \ldots, N u m \_a\right\}$; $C$ is the set of observing chances; $F$ is the set of objective functions. For simplicity, we assume that the number of strips for each orbit is equal, and the satellite can perform 2 imaging tasks (DEM and GMTI) by using the first 3 strips (1,2, and 3$) ; T=\left\{t_{1}, t_{2}, L, t_{m}\right\}$ is the set of observation targets including point and regional targets, where denotes the total number of targets. For each target, we define $t_{i}=\left\{p_{i}\right.$, chance $\left.{ }_{i}\right\}$, where $p_{i}$ is the profit when target $i$ is observed, whose value varies from 1 to 10 , and chance is defined as chance ${ }_{i}\left\{c_{1}, c_{2}, L, c_{n}\right\}$, where $n$ is the total number of observation chances of target $i, c_{i}=\left\{a_{i}, w_{i}, w e_{i}, o_{i}\right\}$, where $a_{i}$ denotes the observation strip and satisfies $a_{i} \in A, w s_{i}$ and $w e{ }_{i}$ stand for start and end times of observation time window, respectively, and $o_{i}$ stands for the sequence number of observation orbit. Apart from the aforementioned variables, there are other parameters defined as follows: 
Time_start: observation mission start time.

Time_end: observation mission end time.

min_ont: the shortest observation time for 1 strip.

max_ont: the shortest observation time for 1 strip.

g_onum: maximum observation numbers in 1 orbit.

g_maxt: the longest observation time in 1 orbit.

time_onf: the shortest time to restart an observation since

the previous observation is stopped.

$b t$ : switching time from one strip to another.

$M g$ : satellite memory storage capacity in 1 orbit.

Num_O: total number of observation time (unit: orbits).

Num_a: total number of observation strips.

$n^{g}$ : total number of strip switches in the $g^{\text {th }}$ orbit.

ont $t_{k}^{g}: k^{\text {th }}$ observing start time in the $g^{\text {th }}$ orbit.

offt ${ }_{k}^{g}: k^{\text {th }}$ observing stop time in the $g^{\text {th }}$ orbit.

After defining the variables used to formulate the multiobjective optimization problem, the multi-objective mission planning problem can be formulated as follows.

\section{OPTIMIZATION VARIABLES}

$x_{i k}= \begin{cases}1 & \text { Target } t_{i} \text { is arranged in the } k^{\text {th }} \text { observation chance } \\ 0 & \text { Target } t_{i} \text { is not implemented }\end{cases}$

$y_{i j}= \begin{cases}1 & \text { when } O_{j}=O_{i}, \text { implemented after } t_{j} \\ 0 & \text { otherwise }\end{cases}$

$x n_{i}= \begin{cases}1 & \begin{array}{l}\text { implemented after } t_{j} \text { and select the current } \\ \text { chance to complete two images }\left(x_{i k}=1, a_{k} \leq 3\right)\end{array} \\ 0 & \text { otherwise }\end{cases}$

\section{OBJECTIVE FUNCTIONS}

$$
\begin{aligned}
& F_{1}=\max \sum_{i=1}^{m} \sum_{k=1}^{l e n} p_{i}\left(x_{i k}\right), \text { len }=\operatorname{length}\left(\text { chance }_{i}\right) \\
& F_{2}=\max \sum_{i=1}^{m} x n_{i}
\end{aligned}
$$

\section{CONSTRAINTS}

$$
\begin{aligned}
& o f f t_{k}^{g}-o n t_{k}^{g} \leq m a x \_o n t \\
& o f f t_{k}^{g}-o n t_{k}^{g} \leq m i n \_o n t \\
& n^{g} \leq g_{\text {onum }}
\end{aligned}
$$

$$
\begin{aligned}
& \sum_{k=1}^{n^{g}}\left(o f f t_{k}^{g}-o n_{k}^{g}\right) \leq g_{\text {maxt }} \\
& w s_{j} \geq w e_{i}+b t+\text { Time }_{\text {start }}, \\
& y_{i j}=1, \text { and } a_{i} \neq a_{j} \\
& o n t_{k}^{g} \leq w s_{i}<w e_{i} \leq o f f t_{k}^{g}, \\
& \text { ont } t_{k+1}^{g} \leq w s_{j}<w e_{j} \leq o f f t_{k+1}^{g} \\
& \text { when }_{i j}=1, O_{i} \neq O_{j} \text { and } y_{j} \geq w e_{i}+\text { time } e_{\text {onf }} \\
& \sum_{k=1}^{\text {len }} x_{i k} \leq 1, \text { Len }=\text { length }\left(\text { chance }_{i}\right)
\end{aligned}
$$

The multi-objective mission planning problem is formulated by using the aforementioned expressions. In details, constraints 6 and 7 indicate that the time of single observation task has the lower and upper bounds. Constraint 8 regulates the longest time of observation in single orbit. Constraint 9 gives the maximum observation number in single orbit. Constraint 10 points out that the time for switching the observation strip from one strip to another has an upper bound. Constraints 11 and 12 present the constraints regarding to the shortest time to restart an observation. Constraint 13 restricts that only 1 observation strip is activated at the same time, and each point target can only be observed once. The optimal objectives contain 2 parts: the first one is the total profit, which is described as objective function (4); the other is the completed image acquisitions expressed by objective function (5). Therefore, the optimal multi-objective mission planning problem can be described as to find the optimal $x_{i k}, x n_{i}$ to maximize $F_{1}, F_{2}$ under the constraints from 6 to 13 .

\section{OPTIMAL SCHEDULING BASED ON NSGA-II ALGORITHM REGIONAL TARGET DECOMPOSITION}

To observe the regional target, we need to divide it into several point targets. Here, we divide the regional target into several squares which have the same longitude and latitude length. The regional target decomposition process is described as follows:

- Step1: determine the maximal and minimal values of the latitude and longitude of the regional target.

- $\quad$ Step 2: after obtaining 4 extreme values from step 1, find the rectangle to surround the regional target, as illustrated in Fig. 3. 
- $\quad$ Step 3: divide the rectangle into several squares based on the latitude and longitude sample intervals.

\section{ENCODING}

Encoding is the key step to apply genetic algorithm. The encoding methods affect the crossover and mutation operator, which determines the efficiency of the genetic evolution. Here, we use the integer coding in which each gene represents a point target whose values range between $0 \sim n$, representing the number of observation opportunities. If the value of this gen is 0 , the corresponding point target has not been observed. As shown in Fig. 4, targets 1 and 140 have not been observed.

\begin{tabular}{|c|c|c|c|c|c|c|c|c|c|c|c|c|c|}
\hline \multicolumn{10}{c|}{ Optimization variables } \\
Chromosome & 0 & 3 & 2 & 2 & 3 & 1 & $\ldots$ & 4 & 2 & 3 & 0 & 1 & 5 \\
Target 1 & Target 3 & Target 6 & $\ldots$ & Target 140 \\
$n_{1}=4$ & $n_{3}=5$ & $n_{5}=4 \quad \ldots$ & $n_{138}=4$
\end{tabular}

Figure 4. The structure of the chromosomes.

\section{CROSSOVER AND MUTATION OPERATOR}

First, we use uniform crossover to select 2 individuals from parent generation by binary tournament, then generate a binary string which has the same length with parent individual randomly, in which 0 represents non-exchange and 1, exchange. Based on the template of binary string to cross 2 parent solutions, new solutions can be obtained, as shown in Fig. 5.

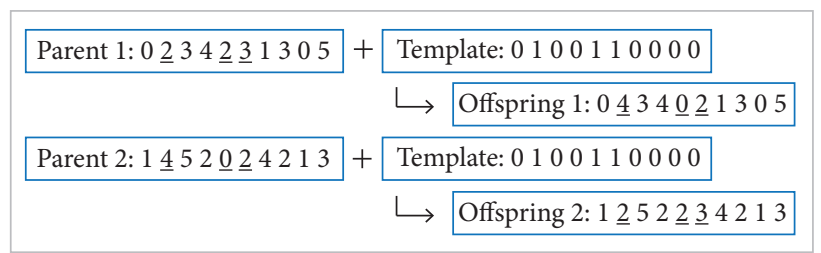

Figure 5. Template of binary string.

The offspring creation process is given by:

- $\quad$ Step 1: select parent solutions $x g_{i}^{(1, l)}$ and $x g_{i}^{(2, l)}$ by binary tournament, where $l$ is the length.

- Step 2: choose a random number $r \in[0,1]$, if $r<P c_{1}$ ( $P c_{1}$ is the crossover probability); go to step 3 , otherwise, non-exchange.

- Step 3: generate a binary string randomly and create offspring solutions $x g_{i}^{(1, l+1)}, x g_{i}^{(2, l+2)}$ by using crossover operator.

We use uniform mutation as mutation operator to improve the local search ability and prevent premature convergence.

\section{ADJUSTMENT SOLUTION CONSIDERING CONSTRAINTS}

Solutions generated by the crossover and mutation processes may not satisfy constraint conditions, hence, these solutions must be filtered to delete unfeasible solutions. For the potential solutions which conflict with the constraints, we change the corresponding gene value of these solutions to 0 (Fig. 6). As shown in Fig. 6, point target 3 does not satisfy constraint conditions, hence, its gene value is changed to 0 .

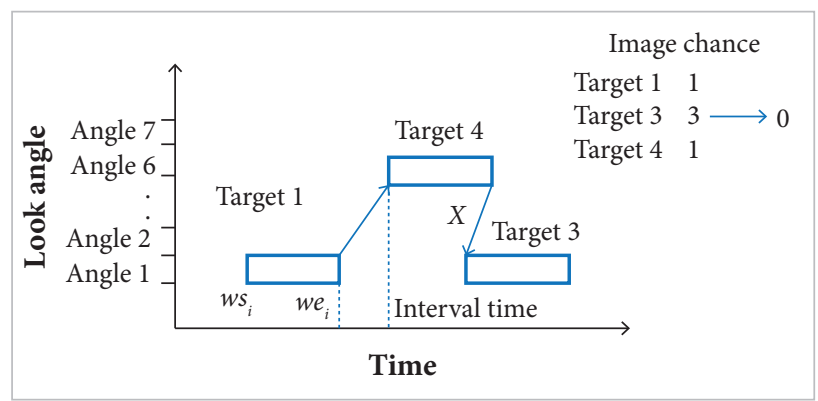

Figure 6. Illustration of observation interval time.

\section{NSGA-II ALGORITHM}

NSGA-II has been demonstrated an efficient algorithm to solve multi-objective optimization problems. Therefore, it has been chosen to solve the proposed multi-objective mission planning optimization problem. Due to the unique feature of this mission planning problem, basic NSGA-II algorithm has been properly modified (Fig.7):

- Step 1: if there are regional targets, they should be decomposed into several point ones.

- Step 2: determine observation opportunity of each target during the mission planning time span.

- $\quad$ Step 3: set proper NSGA-II algorithm parameters, such as population, maximum generation number, crossover, and mutation probabilities.

- $\quad$ Step 4: generate initial population $P_{0}$ randomly.

- Step 5: adjust initial solutions and make them satisfy constraint conditions; evaluate objective functions and perform non-dominated sorting, then calculate crowding distance of individuals and sort them according to crowding distance. Get the first generation $P_{1}$, the generation count number $N_{-}$gen is set to 1 , and record $P_{1}$.

- Step 6: perform uniform crossover and uniform mutation for population $P_{1}$, then obtain offspring generation $Q_{1}$, adjust solution set, and evaluate objective functions (fitness) for individuals in $Q_{1}$. 
- $\quad$ Step7: merge parent population $P_{1}$ and offspring population $Q_{1}$, perform non-dominated sorting, calculate crowding distance between individuals, and sort individuals according to crowding distance in each non-dominated layer.

- Step 8: select individuals which locate on the front and obtain new parent population $P_{2}$.

- $\quad$ Step 9: set $P_{1}$ equals to $P_{2}$, increase generation count number $N_{-}$gen, and record $P_{1}$.

- Step 10: repeat steps from 5 to 8 until $N \_$gen reaches the maximal number.

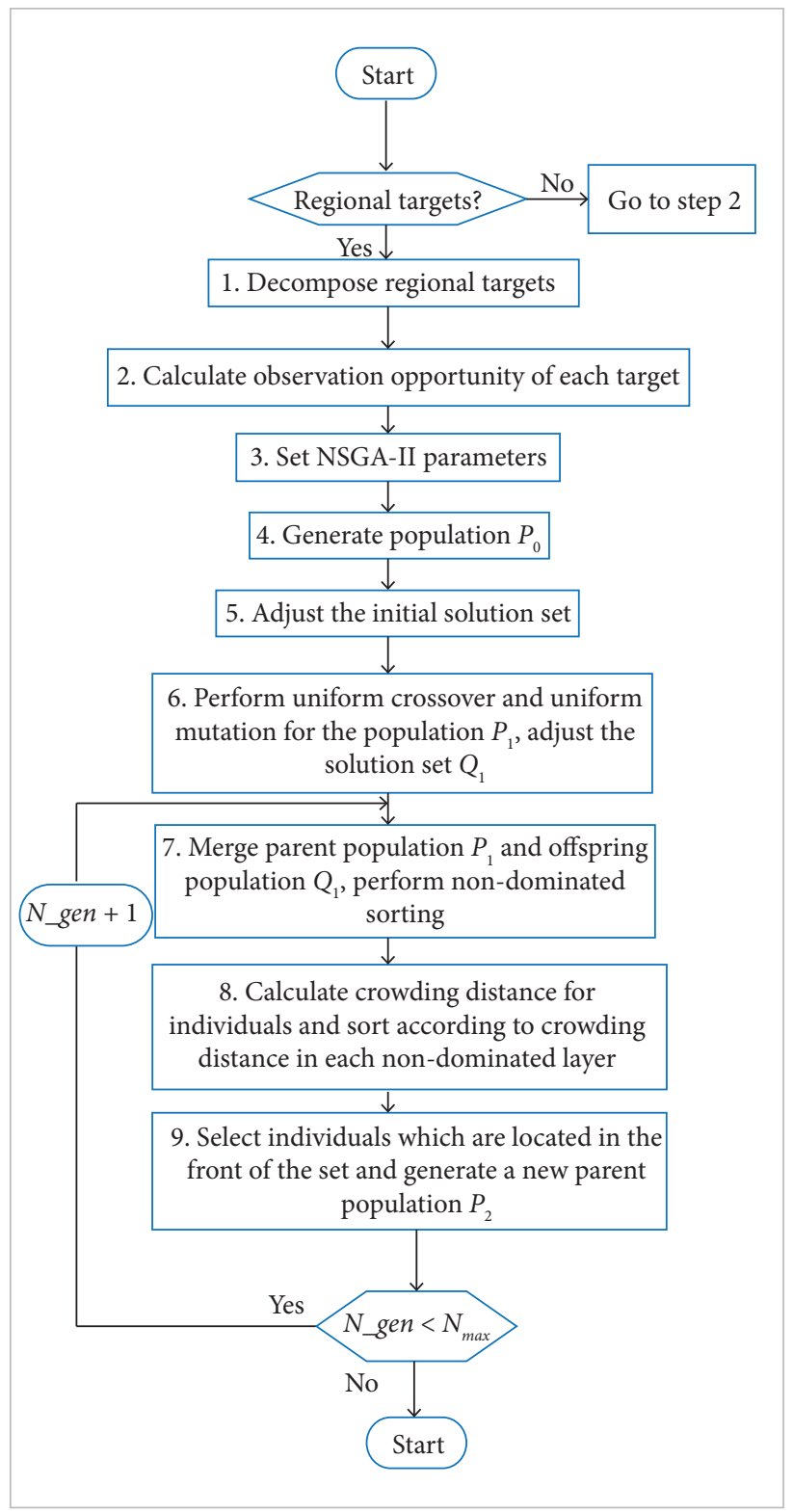

Figure 7. Flowchart for NSGA-II algorithm.

\section{NUMERICAL SIMULATION RESULTS}

In order to verify the validity of the proposed multi-objective mission planning method, several numerical simulations are conducted. The parameters of the numerical simulation are:

- The orbital elements of the chief satellites in the formation are shown in Table 2, where $a$ is orbital semimajor axis, $e$ is eccentricity, $i$ is orbital inclination, $\omega$ is argument of perigee, $\Omega$ is right ascension of ascending node, and $M$ is mean anomaly. The satellite is in Sun synchronous repeating orbit, whose revisit period and orbit repeating cycle are 15 days and 227 orbits, respectively.

- The mission planning start and end times are 2015/07/01 12:00:00 and 2015/07/16 12:00:00, respectively.

- The mission planning mission is conducted, whose point target number is 200 . For single planning mission, the targets are generated and distributed randomly inside Chinese main land (Fig. 8). The priority values of targets vary from 0 to 10 . There is 1 regional target, whose range is $1^{\circ}$ in both latitude and longitude directions, and the sample interval to decompose the regional target is $0.1^{\circ}$ in both latitude and longitude directions. Table 3 presents the point target numbers and the corresponding NSGA-II algorithm parameters.

- The minimal sensor half angle is $19^{\circ}$ and the maximal one is $43^{\circ}$. The whole sensor range is divided into 12 strips (which is abbreviated as st in Table 4). Only the first 3 strips (1,2, and 3 ) of each orbit can perform the 2 tasks (DEM and GMTI) at the same time, which is highlighted in Table 4. The minimal half angles $\left(\alpha^{\text {min }}\right)$ and the maximal ones $\left(\alpha^{\max }\right)$ of these strips are listed in Table 4.

To verify the validity and effectiveness of the proposed multiobjective mission planning optimization method proposed in this study, comparison simulations have been conducted. For the same observation planning missions, 2 different methods are utilized: one is the new proposed NSGA-II method and the other is the general genetic algorithm one. For the latter, 2 different objectives are considered, which are profits and completed image acquisitions.

Table 2. Orbital elements of chief satellite.

\begin{tabular}{|c|c|c|c|c|c|}
\hline $\begin{array}{c}a \\
(\mathrm{~km})\end{array}$ & $e$ & $\begin{array}{c}i \\
\left.{ }^{\circ}\right]\end{array}$ & $\begin{array}{c}\omega \\
\left({ }^{\circ}\right)\end{array}$ & $\begin{array}{c}\Omega \\
\left({ }^{\circ}\right)\end{array}$ & $\begin{array}{c}M \\
\left({ }^{\circ}\right)\end{array}$ \\
\hline $6,898.224109$ & 0.001075 & 97.482440 & 0 & 9.312805 & 180 \\
\hline
\end{tabular}


Table 3. Simulation parameters of scenario 1.

\begin{tabular}{|c|c|c|c|c|c|}
\hline Scenario & Target numbers & N_pop & N_top & Crossover probability & Mutation probability \\
\hline 1 & 200 & 100 & 1,200 & 0.9 & 0.1 \\
\hline
\end{tabular}

Table 4. Inner and outer half angles of different strips.

\begin{tabular}{|c|c|c|c|c|c|c|c|c|c|c|c|c|}
\hline & st 1 & st 2 & st 3 & st 4 & st 5 & st 6 & st 7 & st 8 & st 9 & st 10 & st 11 & st 12 \\
\hline$a^{\min }\left({ }^{\circ}\right)$ & 19.7 & 22.3 & 24.8 & 27.2 & 29.5 & 31.8 & 33.9 & 36.0 & 37.9 & 39.8 & 41.6 & 43.4 \\
\hline$\alpha^{\max }\left(^{\circ}\right)$ & 23.1 & 25.6 & 27.9 & 30.2 & 32.4 & 34.6 & 36.6 & 38.5 & 40.4 & 42.2 & 43.9 & 45.5 \\
\hline
\end{tabular}

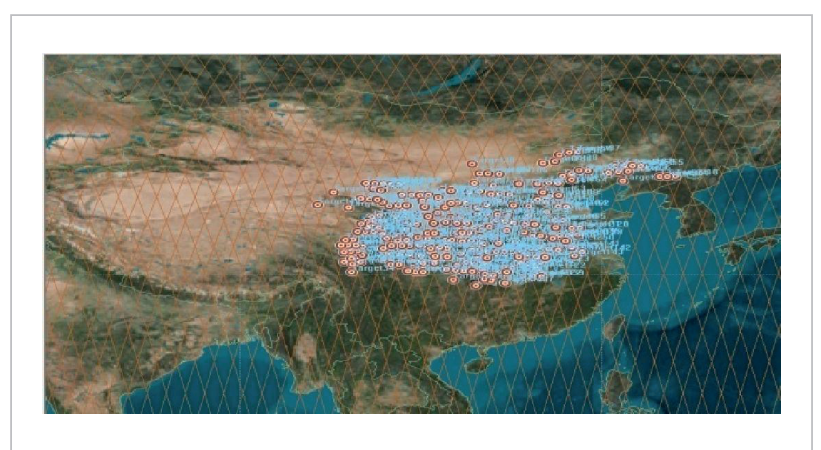

Figure 8. Distribution of observation targets in the map.

For target number equal to 200, Fig. 9 shows the results of the proposed NSGA-II method and of the general algorithm one. The 3 lines in Figs. 9a and 9b show the total profits and completed image acquisitions of the NSGA-II method, as well as genetic algorithm method with total profits as the optimization function (abbreviated as GA_Income) and genetic algorithm method with total completed image acquisitions as the optimization function (abbreviated as GA_Num). In Fig. 9a, the green line shows the profit values by using genetic algorithm method with the total profits as the optimization function, the blue line indicates the profit value by using genetic algorithm method with the completed image acquisitions as the optimization function, and the red line shows the profit values by utilizing NSGA-II methods. Figure 9a shows that the total profit values obtained by GA_Income method are larger than those of the proposed NSGA_II method, which are larger than those of the GA_Num method. Figure 9b shows that the total completed image acquisitions obtained by GA_Num method are larger than those of the proposed NSGA_II method, which are larger than those of the GA_ Income method. From Figs. 9a and 9b, the conclusion is that, by using the proposed NSGA-II method, although the single objective value is less than the values obtained by GA_Num

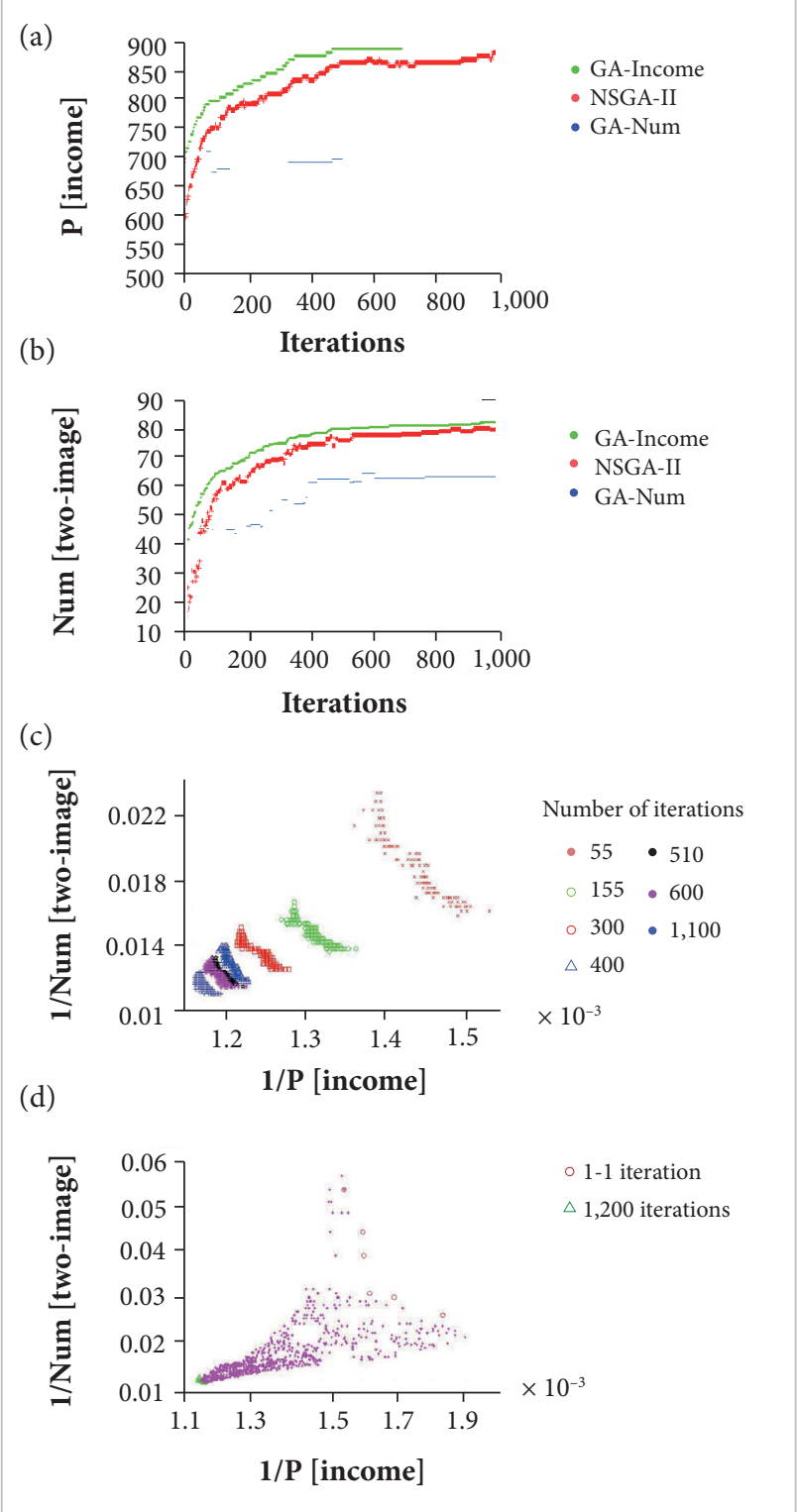

Figure 9. Multi-objective mission planning results (targets number equal to 200). 
or GA_Income methods, one can obtain better total profits and completed image acquisitions objectives in an multiobjective optimization sense. Figures $9 \mathrm{c}$ and $9 \mathrm{~d}$ demonstrate that the proposed NSGA-II method is converged after 1,200 iterations. In Fig. 9d, the pink indication means that the middle interactions show the coverage process of solution.

\section{CONCLUSION}

In this study, a NSGA-II-based multi-objective mission planning optimization method is proposed to deal with the unique SAR formation flying image mission planning problem, whose unique feature is that the formation system can perform 2 different tasks (DEM and GMTI) at the same time compared to the traditional single satellite mission planning problem, which can perform only a single task. The 2 different objective functions are defined as total profits and completed image acquisitions numbers, respectively. Several practical constraints are firstly considered when formulating the multi-objective mission planning problem, such as the longest observation time in 1 orbit and strip switching time from one strip to another.
Numerical simulations show that, although the single objective value is less than the values obtained by genetic algorithm method with single objective function, the proposed multiobjective mission planning optimization method can obtain better performance with respect to 2 mission planning objectives in an overall perspective.

\section{ACKNOWLEDGEMENTS}

The authors are grateful for the support provided for this study by the National Natural Science Foundation of China (No. 11502142).

\section{AUTHOR'S CONTRIBUTION}

Shao $\mathrm{X}$ and Zhang $\mathrm{Z}$ conceived the idea and co-wrote the main text; Shao X, Zhang Z, and Wang J performed the numerical simulation and analyzed the results; Zhang D prepared the tables and figures. All authors discussed the results and commented on the manuscript.

\section{REFERENCES}

Bensanna E, Verfaillie G, Agnèse JC, Bataille N, Blumstein D (1996) Exact and inexact methods for the daily management of an earth observation satellite. Proceedings of the 4th International Symposium on Space Mission Operations and Ground Data Systems; Munich, Germany.

Bianchessi N, Cordeau JF, Desrosiers J, Laporte G, Raymond V (2007) A heuristic for the multi-satellite, multi-orbit and multiuser management of Earth observation satellites. Eur J Oper Res 177(2):750-762.doi: 10.1016/j.ejor.2005.12.026

Gao P, Tan YJ, Li JF, He RJ (2013) An ant colony algorithm for remote satellite and ground integration scheduling problem in parallel environment. Adv Mater Res 791:1341-1346. doi: 10.4028/www.scientific.net/AMR.791-793.1341

Hao HC, Jiang W, Li YJ (2013) Mission planning for agile earth observation satellites based on hybrid genetic algorithm. Science Technology and Engineering 13(17):4972-4978.

He RJ, Gao P, Bai BC (2011) Models, algorithms and applications to the mission planning systems of imaging satellites. Systems
Engineering - Theory and Practice 31(3):411-422.

Jang J, Choi J, Bae HJ, Choi IC (2013) Image collection planning for KOrea Multi-Purpose SATellite-2. Eur J Oper Res 230(1):190-199. doi: 10.1016/j.ejor.2013.04.009

Kim H, Chang YK (2015) Mission scheduling optimization of SAR satellite constellation for minimizing system response time. Aero Sci Tech 40:17-32. doi: 10.1016/j.ast.2014.10.006

Lemaitre M, Verfaillie G, Jouhaud F, Lachiver JM, Bataille N (2002) Selecting and scheduling observations of agile satellites. Aero Sci Tech 6(5):367-381. doi: 10.1016/S1270-9638(02)01173-2

Tangpattanakul P, Jozefowiez N, Lopez P (2015) A multi-objective local search heuristic for scheduling Earth observations taken by an agile satellite. Eur J Oper Res 245(2):542-554. doi: 10.1016/j. ejor.2015.03.011

Xiaolu L, Baocun B, Yingwu C, Feng Y (2014) Multi satellites scheduling algorithm based on task merging mechanism. Appl Math Comput 230:687-700. doi: 10.1016/j.amc.2013.12.109 